\title{
EphB Receptors Regulate Stem/Progenitor Cell Proliferation, Migration, and Polarity during Hippocampal Neurogenesis
}

\author{
Michael J. Chumley, ${ }^{1}$ Timothy Catchpole, ${ }^{1}$ Robert E. Silvany, ${ }^{1}$ Steven G. Kernie, ${ }^{1,2}$ and Mark Henkemeyer ${ }^{1}$ \\ Departments of ${ }^{1}$ Developmental Biology and ${ }^{2}$ Pediatrics, University of Texas Southwestern Medical Center, Dallas, Texas 75390
}

\begin{abstract}
The adult brain maintains two regions of neurogenesis from which new neurons are born, migrate to their appropriate location, and become incorporated into the circuitry of the CNS. One of these, the subgranular zone of the hippocampal dentate gyrus, is of primary interest because of the role of this region in learning and memory. We show that mice lacking EphB1, and more profoundly EphB1 and EphB2, have significantly fewer neural progenitors in the hippocampus. Furthermore, other aspects of neurogenesis, such as polarity, cell positioning, and proliferation are disrupted in animals lacking the EphB1 receptor or its cognate ephrin-B3 ligand. Our data strongly suggest that EphB1 and ephrin-B3 cooperatively regulate the proliferation and migration of neural progenitors in the hippocampus and should be added to a short list of candidate target molecules for modulating the production and integration of new neurons as a treatment for neurodegenerative diseases or brain injury.
\end{abstract}

Key words: hippocampus; neurogenesis; dentate gyrus; Eph receptor tyrosine kinase; ephrin ligand; cell polarity

\section{Introduction}

The hippocampus is a unique structure in that it is one of the few regions of the brain capable of producing new neurons well into adulthood (Lennington et al., 2003; Abrous et al., 2005; Ming and Song, 2005; Lledo et al., 2006). New neurons are continuously generated in the subgranular zone (SGZ) of the hippocampal dentate gyrus (DG), migrate only a short distance into the granule layer (GL), and terminally differentiate mainly into new dentate granule cell neurons. The newly generated granule cell neurons elaborate thick dendritic branches into the molecular layer (ML) of the DG and begin receiving synaptic inputs from perforant path afferents while extending mossy fiber track axons into the $\mathrm{CA} 3$ region to complete integration into the existing neural circuitry (Esposito et al., 2005; Song et al., 2005; Tozuka et al., 2005; Wang and Chia, 2005; Ge et al., 2006; Overstreet-Wadiche and Westbrook, 2006). It has only recently been demonstrated that these new adult-born neurons subsequently integrate into memory networks (Kee et al., 2007). Considering that the hippocampus is involved in encoding long-term episodic memories and in spatial navigation (Squire, 1992; Holscher, 2003; Suzuki, 2006) as well as regulating memories of personally experienced events and their associated emotions (Phelps, 2004; Richardson et al., 2004), it is not surprising that devastating insult to the brain leading to hippocampal damage has serious clinical consequences and is an important area of stem cell research.

\footnotetext{
Received May 16, 2007; revised 0ct. 18, 2007; accepted 0ct. 22, 2007.

This work was supported by National Institutes of Health Grants R01 NS048192 (S.G.K.) and R01 MH066332 (M.H.). We thank Tracey Bowdler and Jan La for genotyping and Shawna Kennedy for flow cytometry. Special thanks to Angie Mobley for FACS sorting.

Correspondence should be addressed to Dr. Mark Henkemeyer at the above address. E-mail: mark.henkemeyer@utsouthwestern.edu.

DOI:10.1523/JNEUROSCI.4158-07.2007

Copyright $\odot 2007$ Society for Neuroscience $\quad$ 0270-6474/07/2713481-10\$15.00/0
}

Two groups of cell-surface molecules that are involved in many aspects of cell migration, axon guidance, and synaptogenesis are the Eph receptor tyrosine kinases and their ephrin ligands. Eph receptors are divided into two separate subfamilies, termed EphA and EphB, and contain cytoplasmic tyrosine kinase catalytic domains that are activated after interactions with their cognate cell surface-bound ephrin ligands. Each EphB receptor can promiscuously bind to any B-subclass ephrin, but weakly or not at all to A-subclass ephrins (Davis et al., 1994; Brambilla et al., 1995; Gale et al., 1996), with just one exception (Himanen et al., 2004). After engagement of the EphB receptors with their ephrin-B ligands at the interface of cell-cell contact, both the receptor and ligand transduce intracellular signals in a bidirectional manner into the receptor-expressing cell (forward signaling) and ephrin-expressing cell (reverse signaling) (Henkemeyer et al., 1996; Holland et al., 1996). In general, Eph-ephrin bidirectional signaling is thought to converge on the cytoskeleton to ultimately induce changes in cell/axon migration that in most described instances result in repulsive events, such as axonal growth cone collapse, although roles in cell-cell adhesion and axonal attraction have been reported (Holmberg and Frisen, 2002). In vivo roles for Eph-ephrin bidirectional signaling in cell proliferation have not been widely recognized, although a small number of reports have implicated activities in stem/progenitor cells (Conover et al., 2000; Depaepe et al., 2005; Holmberg et al., 2006; Ricard et al., 2006). However, functions for Ephs and ephrins in hippocampal stem/progenitor cells have not previously been appreciated, even though these molecules are well known to regulate synaptic functions in the hippocampus. Here we demonstrate that EphB receptors are specifically expressed in hippocampal stem/progenitor cells of the dentate gyrus and have key in vivo roles in regulating the proliferation and migration of these cells by interacting with the ligand ephrin-B3. 


\section{Materials and Methods}

Mice. EphB1 (Williams et al., 2003), EphB2 (Henkemeyer et al., 1996), and ephrin-B3 (Yokoyama et al., 2001) mutant animals and nestin-eGFP (Yu et al., 2005) transgenic animals, all on the CD1 background, were housed in the Animal Resource Center at the University of Texas Southwestern Medical Center at Dallas. All experiments on animals were conducted according to protocols approved by the Institutional Animal Care and Use Committee.

Immunohistochemistry and immunofluorescence. All immunostaining was performed on 8- to 10-week-old adult mice unless otherwise stated. Mice were deeply anesthetized using ketamine/xylazine and were transcardially perfused with $4 \%$ paraformaldehyde (PFA) in PBS. Brains were removed and fixed overnight in $4 \%$ PFA. Coronal vibratome sections ( 40 $\mu \mathrm{m})$ from agarose-embedded brains were collected and stored in $1 \%$ PFA at $4^{\circ} \mathrm{C}$. Sections from the rostralmost complete DG to the most caudal section with a DG were counted, and four to six equally spaced sections were selected from each animal for immunohistochemistry. Rabbit anti-green fluorescent protein (GFP) (Invitrogen, Carlsbad, CA), rabbit anti-Ki-67 (NeoMarkers, Fremont, CA), goat anti-doublecortin (DCX; Santa Cruz Biotechnology, Santa Cruz, CA), rat anti- $\beta$ galactosidase ( $\beta$-gal), mouse anti-polysialic acid (PSA)-neural cell adhesion molecule (NCAM), and mouse anti-NeuN (all from Millipore, Billerica, MA) were incubated with floating sections overnight at $4^{\circ} \mathrm{C}$. Appropriate donkey secondary antibodies with $\mathrm{Cy} 2, \mathrm{Cy} 3$, or $\mathrm{Cy} 5$ conjugates were also incubated with floating sections overnight at $4^{\circ} \mathrm{C}$. Toto- 3 (Invitrogen) was used to label DNA were indicated. Confocal images were obtained on a Zeiss (Thornwood, NY) LSM-510. Image analysis was performed using ImageJ software (http://rsb.info.nih.gov/ij/).

5-Bromo-4-chloro-3-indolyl- $\beta$-D-galactopyranoside (X-gal) staining procedures have been previously described (Henkemeyer et al., 1996). Briefly, fresh brains were isolated from $\mathrm{CO}_{2}$-killed animals and frozen in JUNG Tissue Freezing Medium (Leica Microsystems, Bannockburn, IL) and immediately cryosectioned on a Leica CM3050S cryostat (Leica Microsystems). Sections were postfixed on slides in 4\% PFA and incubated overnight at $37^{\circ} \mathrm{C}$ in $\mathrm{X}$-gal solution. Slides were counterstained in $\mathrm{Nu}-$ clear Fast Red solution (Polysciences, Warrington, PA) and color images obtained with a Nikon (Tokyo, Japan) DMX-1200 color digital camera on an Olympus (Tokyo, Japan) BX-50 microscope.

To carry out double labeling for calretinin and ephrin-B3 expression, brains from $4 \%$ PFA perfused ephrin-B $3^{\text {lacZ/lacZ }}$ animals were vibratome sectioned, washed, and processed overnight in X-gal for detection of the ephrin-B3- $\beta$-gal fusion protein. After subsequent washing to remove excess X-gal, sections were postfixed in $4 \%$ PFA for $15 \mathrm{~min}$, washed, and processed for immunohistochemistry using rabbit anti-calretinin antibodies (Millipore), biotinylated donkey anti-rabbit IgG (Jackson ImmunoResearch, West Grove, PA), and streptavidin-horseradish peroxidase (Pierce, Rockford, IL) with the DAB-Plus kit (Zymed, South San Francisco, CA) to label calretinin-positive mossy cells in the DG. Alternatively, perfused, vibratome-sectioned brains were processed for immunofluorescence using anti- $\beta$-gal and anti-calretinin antibodies as described above.

Bromodeoxyuridine administration. Single intraperitoneal injections $(150 \mathrm{mg} / \mathrm{kg})$ or a series of three injections separated by $2 \mathrm{~h}(50 \mathrm{mg} / \mathrm{kg}$ each) were used to study proliferation in the DG. Bromodeoxyuridine (BrdU; Sigma, St. Louis, MO) was dissolved in PBS at $10 \mathrm{mg} / \mathrm{ml}$ and administered sequentially in animals so that all times until the animals were killed were equal in each experimental group. In experiments with a single injection, animals were killed $2 \mathrm{~h}$ after BrdU administration. In experiments with multiple injections per animal, groups of animals were killed at 2 or $24 \mathrm{~h}$ or at 7, 14, or $28 \mathrm{~d}$ after BrdU administration.

Cell counting. $\mathrm{GFP}^{+}, \mathrm{BrdU}{ }^{+}$, or $\mathrm{Ki}-67^{+}$cells were counted in equivalent coronal vibratome sections. The entire DG region on each hippocampus was imaged as a $30 \mu \mathrm{m}$-thick $z$-series, and individual planes were analyzed to differentiate individual cells in clusters. Total cell counts were obtained, and positions of the individual cells within the DG were recorded. All data analysis was blinded to genotype. Statistical analysis was performed using Student's $t$ test or ANOVA and appropriate post hoc comparisons as noted.
Dendrite counting. After immunolabeling for DCX in equivalent coronal sections, a $30-\mu \mathrm{m}$-thick $z$-series of confocal images was collected at several points along the GL. Separate regions of interest within the GL and perpendicular to the radially projecting $\mathrm{DCX}^{+}$dendrites were selected through the $z$-plane, and a voxel count was performed. This process was repeated at three equally spaced $z$-planes in the ML. All analysis was performed using ImageJ and appropriate plug-ins.

Volume determination of the DG. For DG volume determination, every fifth vibratome section $(40 \mu \mathrm{m})$ was Nissl stained and imaged. Using ImageJ software, the DG was outlined, and the area was obtained. Volume was estimated using the formula $\sum A \times T \times I \times 2$, where $\sum A$ is the sum of the area measured on each section, $T$ is the section thickness, $I$ is the number of section intervals, and 2 accounts for two half brains. The number of sampling points (sections) varied between genotypes; thus, sampling began at the first indication of a complete DG and ended when the DG was no longer visible. Mean volumes for all genotypes sampled were compared using ANOVA. Preplanned comparisons between wildtype and each EphB mutant mean volume were performed using a Bonferroni correction.

Fluorescence-activated cell sorting. Animals at postnatal days 7 and 14 were killed with ketamine/xylazine, and the hippocampus was removed from the brain. Each side of the hippocampus was cut into $500 \mu \mathrm{m}$ sections perpendicular to the long axis using a McIlwain tissue chopper. Sections were then flattened in DMEM/F-12 (Mediatech, Manassas, VA) supplemented with $10 \%$ FCS, and the DG was microdissected and removed. DG tissues were then digested in the papain dissociation system (Worthington Biochemical, Lakewood, NJ) and resuspended in DMEM/ F-12 supplemented with 10\% FCS. Cell suspensions were immunolabeled using a mouse anti-PSA-NCAM antibody (Millipore) and tagged with a Cy5-conjugated secondary antibody before being washed and resuspended in $10 \mathrm{~mm}$ HEPES-buffered PBS with 2\% FCS. Cell suspensions were filtered through a $70 \mu \mathrm{m}$ cell strainer (BD BioSciences, San Jose, CA) before sorting on a Mo-Flo (DakoCytomation, High Wycombe, UK). In separate experiments, analysis of cell populations was performed on a FACSAria (BD Biosciences). All postcytometric data analysis was performed using FlowJo software (Tree Star, Ashland, OR).

$R N A$ extraction and reverse transcription-PCR. Total RNA was isolated from fluorescence-activated cell sorting (FACS)-sorted cell populations using the MasterPure RNA purification kit (EPICENTRE Biotechnologies, Madison, WI), and first-strand cDNA synthesis was performed using the MonsterScript first-strand cDNA synthesis kit (EPICENTRE Biotechnologies) and supplied oligo(dT) primers. PCR amplification of first-strand cDNA was performed with the following oligonucleotides: EphB1-forward (5'-AAGCCCCCTACCTCAAAGTG-3') and EphB1reverse (5'-CACCATCCACTCCATCTCCG-3') producing a $353 \mathrm{bp}$ band, EphB2-forward (5'-TAGTCTTCCTCATCGCTGTG-3') and EphB2-reverse (5'-ATGATGGATGCCTCACTCAG-3') producing a 366 bp band, ephrin-B3-forward (5'-CCCCAAACCTTCTTCTCACA$\left.3^{\prime}\right)$ and ephrin-B3-reverse (5'-ACTCGCAGAAGCACCTTCAT- $\left.3^{\prime}\right)$ producing a 212 bp band, glyceraldehyde-3-phosphate dehydrogenase (GAPDH)-forward (5' GACCCCTTCATTGACCTCAACTACATG-3') and GAPDH-reverse (5'-GTCCACCACCCTGTTGCTGTAGCC- $\left.3^{\prime}\right)$ producing a $453 \mathrm{bp}$ band, and GFP-forward (5'-TACGGAAAGCTGACCCTGAAGT- $3^{\prime}$ ) and GFP-reverse (5'-CGTCCTTGAAGAAGATGGTGCG-3') producing a 190 bp band.

\section{Results}

EphB1 is expressed in neural stem and progenitor cells in the adult dentate gyrus

We first addressed the expression of the EphB1 receptor in the adult hippocampus by staining brain sections of 4 -week-old $E p h B 1^{\text {lacZl+ }}$ mutant mice with X-gal to detect the knocked-in lacZ/ $\beta$-gal reporter. Previous studies have shown that this $E p h B 1^{\text {lacZ }}$ mutation provides a high signal-to-noise ratio reporter that faithfully marks the cells that normally express EphB1 with cytoplasmic $\beta$-gal (Williams et al., 2003). Within the dentate region of the hippocampus, distinct $\beta$-gal-stained cells were observed in the SGZ where proliferative self-renewing "stem" and 
a

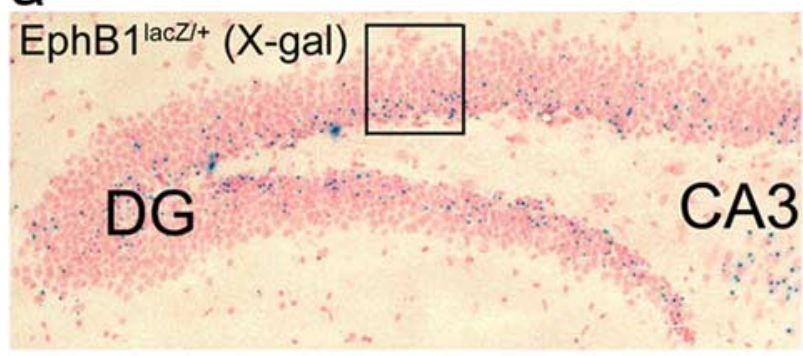

b

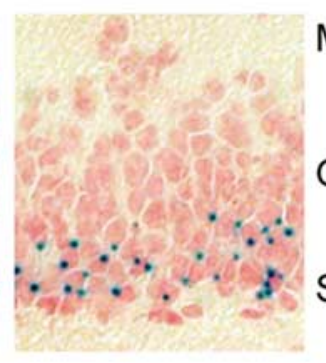

ML

GL

SGZ

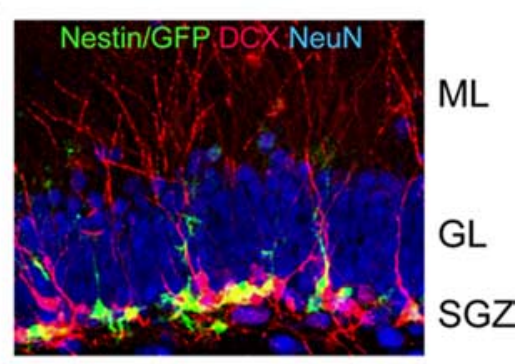

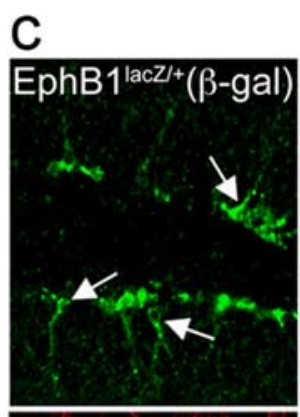

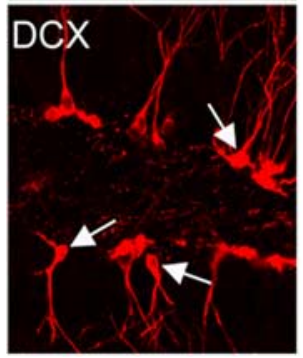

d

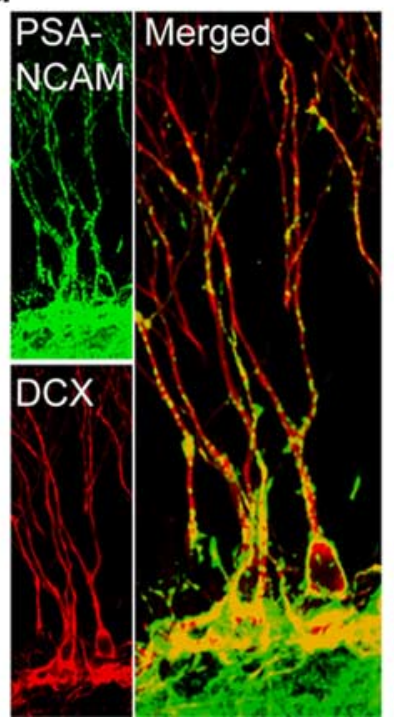

e

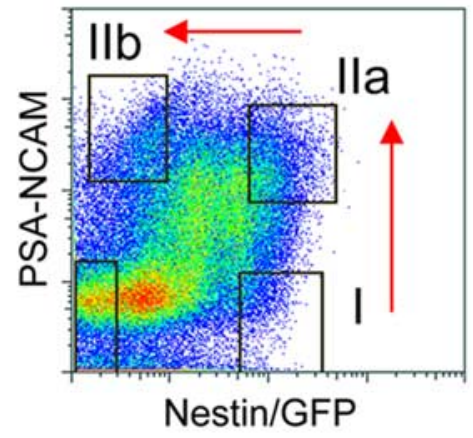

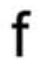

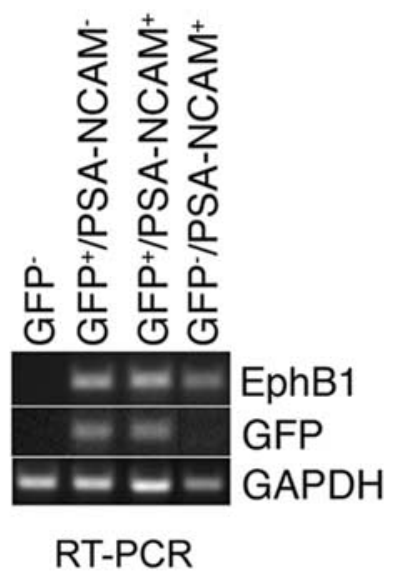

Figure 1. EphB1 is expressed in the adult hippocampal neural stem/progenitor cell population. $\boldsymbol{a}$, Low- and high-magnification bright-field coronal images of the DG from a 4-week-old EphB $1^{\text {lacZ/+ }}$ mouse reacted with X-gal shows expression of the knocked-in $\beta$-gal reporter (blue stain) in the SGZ. $b$, Confocal IF labeling of an 8-to 10-week-old adult DG from nestin-eGFP transgenic reporter mice using GFP (green), DCX (red), and NeuN (blue) antibodies identifies type I (green), type lla (yellow), and type llb (red) stem/progenitor cells in the SGZ and mature neurons (blue) in the GL. c, Confocal IF of an 8- to 10-week-old EphB $1^{\text {lacZ/+ }}$ adult using $\beta$-gal (green) and DCX (red) antibodies demonstrates coexpression of EphB1 in DCX ${ }^{+}$cells within the SGZ (arrows).d, Confocal IF using DCX (red) and PSA-NCAM (green) antibodies demonstrates coexpression of both markers on adult stem/progenitors (yellow). $e$, Flow cytometry (FACS) of cells isolated from nestin-eGFP transgenic mice after microdissection of the DG from 2-week-old animals and sorted for eGFP fluorescence and PSA-NCAM immunoreactivity. Arrows represent developmental progression of the neural progenitor cells, and boxes represent sorted populations. All sorted populations resulted in $\geq 95 \%$ purity after postsort analysis. $f$, RT-PCR from total RNA collected from sorted populations in e detects EphB1 transcripts in all three neural progenitor groups but not in the nonprogenitor population (GFP ${ }^{-} /$PSA-NCAM $^{-}$).

transient-amplifying "progenitor" cells reside (Fig. 1a). A search of the Allen Brain Atlas database of mRNA in situ (www. brainatlas.org) (Lein et al., 2007) provided a similar SGZ pattern for EphB1 transcripts (supplemental Fig. 1a, available at www. jneurosci.org as supplemental material).

To better characterize EphB1 expression in the SGZ, we used a transgenic mouse that expresses enhanced green fluorescent protein (eGFP) under the control of select elements of the nestin promoter. This nestin-eGFP transgene has been shown to mark the earliest stem/progenitor populations in the adult SGZ of the hippocampus with eGFP (Fig. 1b) (Yu et al., 2005). The birth of new neurons in the SGZ progresses from nestin ${ }^{+}$type I stem cells that coexpress the glial cell marker GFAP, to progenitor cells that coexpress nestin and the microtubule-associated protein DCX (type IIa), to progenitor cells that only express DCX (type IIb), to, finally, mature neurons that express the marker NeuN (Fig. 1b). Double immunofluorescence (IF) of EphB1 $1^{\text {lacZ/+ }}$ mice with anti$\beta$-gal and anti-DCX antibodies identifies most EphB1-expressing cells as $\mathrm{DCX}^{+}$type IIa and type IIb neuronal progenitors (Fig. 1c). Because DCX is an intracellular molecule, we sought to find a cell-surface protein that could identify the $\mathrm{DCX}^{+}$type IIa and type IIb progenitors and be used to sort these cell populations by
FACS. In our hands, the cell surface PSA modification that occurs on the NCAM overlaps with DCX expression at close to $100 \%$ (Fig. 1d). Using eGFP fluorescence driven by the nestin-eGFP transgene and anti-PSA-NCAM antibodies, we were able to FACS sort the cells representative of the three different types of stem/progenitor cells from microdissected DG obtained from 2-week-old mice (Fig. 1e). Reverse transcription (RT)-PCR analysis of total RNA isolated from the various sorted cell populations detected EphB1 transcripts in all three neural stem/progenitor cell types, but not in the more differentiated nestin/eGFP ${ }^{-}$and PSA-NCAM $^{-}$cells (Fig. $1 f$ ). In summary, our data provide strong evidence and are the first to document that EphB1 is expressed on proliferating stem/progenitor cells in the hippocampus.

EphB1 regulates progenitor cell number, proliferation, and positioning in the DG

To investigate the possible roles for EphB1 receptors in hippocampal neurogenesis, the $E p h B 1^{-}$protein null allele that does not contain inserted lac $Z$ sequences (Williams et al., 2003) was crossed to the nestin-eGFP transgenic mouse to mark type I and type IIa stem/progenitor cells in resulting EphB1 $1^{-1-}$ mutants 
a
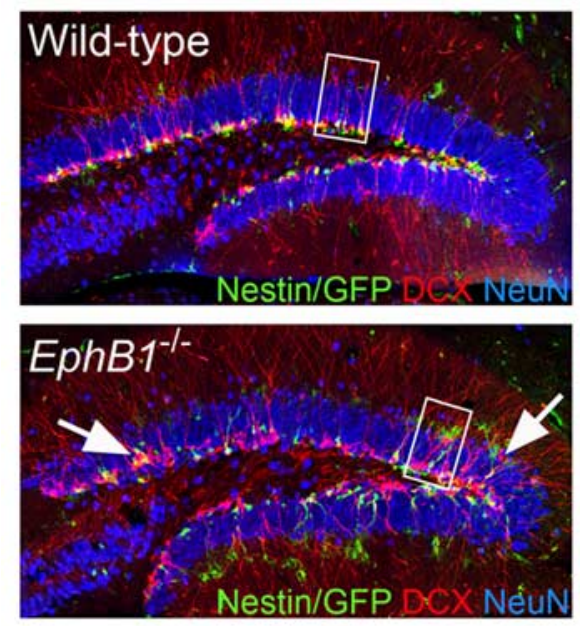

b
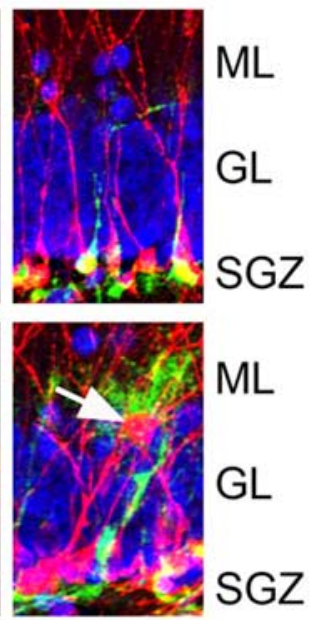

Wild-type $(n=12)$

EphB1 ${ }^{--}(\mathrm{n}=12)$

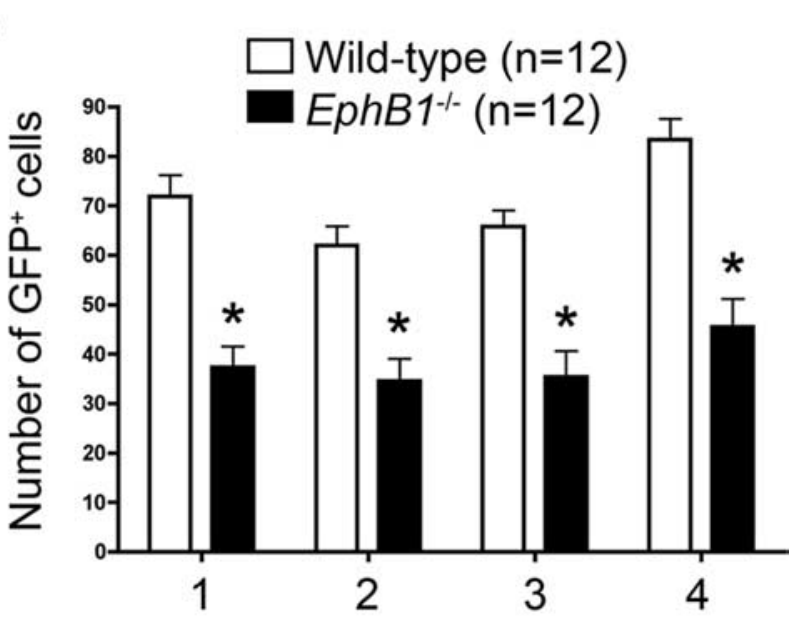

C
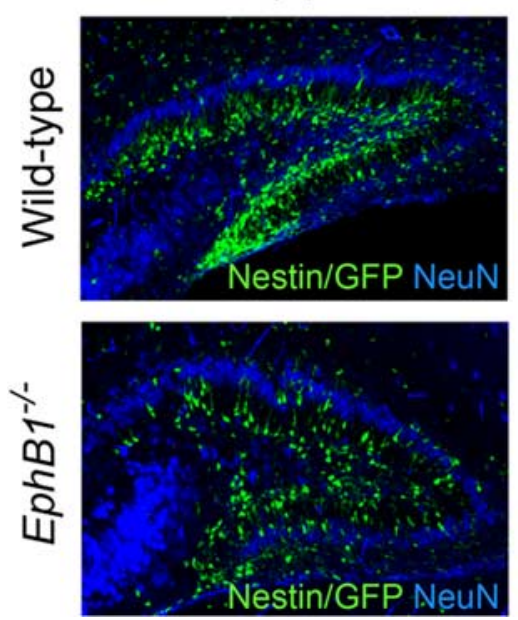

P14
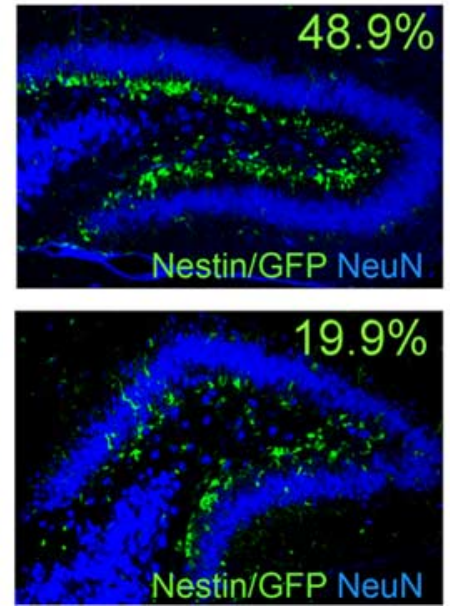

\section{Rostral-caudal position}


a
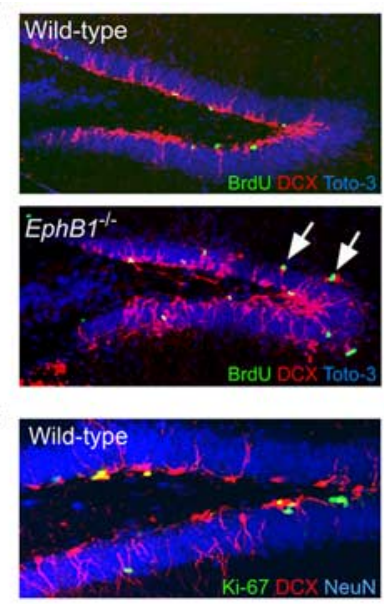

b
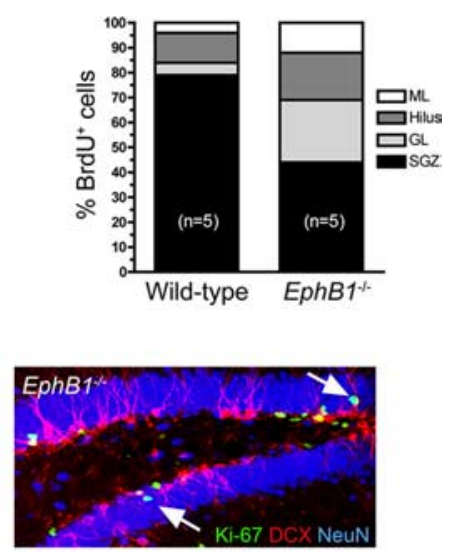

d

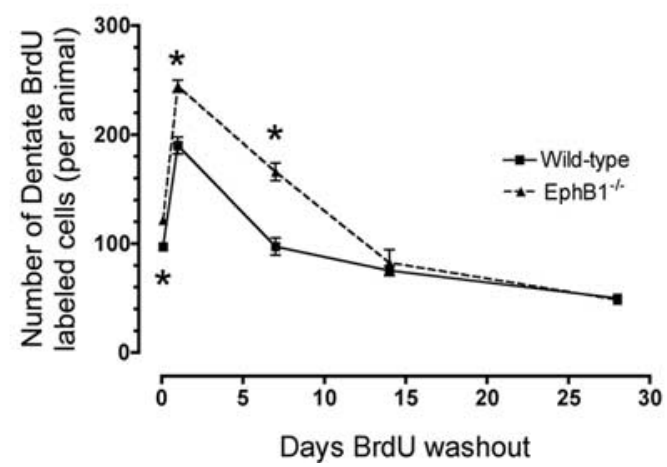

Figure 3. Ectopic localization of proliferating cells in the DG of mice lacking EphB1. $\boldsymbol{a}, A$ single intraperitoneal injection of BrdU was used to label proliferating cells in 8- to 10-week-old mice. After a $2 \mathrm{~h}$ washout, hippocampi were collected and processed for confocal IF to detect cells that incorporated BrdU (green) and expressed DCX (red). In wild-type animals, most proliferating cells are $D C X^{+}$and are localized in a tight band of cells that comprise the $S G Z$, whereas in EphB1 ${ }^{-1-}$ mutants, numerous proliferating cells are detected outside of the SGZ (arrows). The ectopic proliferating cells in EphB1 ${ }^{-1-}$ mutants are often $\mathrm{DCX}{ }^{+}$or found in close association with $D C X^{+}$cells, suggesting active sites of neurogenesis. All DNA is labeled with TOTO-3 (blue). $\boldsymbol{b}$, Quantitative cell counts were compared using Student's $t$ test and indicate that EphB1 ${ }^{-1-}$ animals have a significantly lower percentage of total proliferating $\left(\mathrm{BrdU}^{+}\right)$cells in the SGZ ( $p=0.0002)$ and have a significantly higher percentage in the $\mathrm{GL}(p=0.0078), \mathrm{H}$ $(p=0.0376)$, and ML ( $p=0.0048)$. c, Confocal IF for Ki-67 (green) also detects proliferating cells outside of the SGZ and in close association with mislocalized DCX ${ }^{+}$cells (red) in the EphB1 ${ }^{-1-}$ mutants. $\boldsymbol{d}$, BrdU "birth dating" suggests more proliferating cells in the DG of EphB1 ${ }^{-1-}$ mutants. Three injections of BrdU separated by $2 \mathrm{~h}$ were used to label a substantial portion of cells in S-phase. Comparisons of total BrdU ${ }^{+}$cells were performed after 2 and $24 \mathrm{~h}$ washouts as well as 7, 14, and $28 \mathrm{~d}$ after the last BrdU injection. The total number of BrdU ${ }^{+}$cells is presented for six coronal sections from each animal. A Student's $t$ test indicates that EphB1 ${ }^{-1-}$ animals have significantly more cells in S-phase at 2 and $24 \mathrm{~h}$, as well as $7 \mathrm{~d}$ after BrdU administration ( ${ }^{*} p<0.05$ ). Error bars represent \pm SEM for $n=3-7$ animals per genotype at each time point.

In support of a possible migration defect, labeling for nestin/ eGFP and DCX also identified ectopic stem and progenitor cell bodies outside of the SGZ in the EphB1 $1^{-/-}$mutants, often located deep within the GL as well as in the ML and hilus $(\mathrm{H})$ of the DG (Fig. 2a). To determine whether these ectopic progenitors were actively involved in neurogenesis, we used a single BrdU injection and $2 \mathrm{~h}$ washout to assess for cell proliferation and demonstrated that there is a shift of proliferation in the adult dentate from the SGZ of wild-type animals to the GL and outside the GL in EphB1 ${ }^{-1-}$ animals (Fig. 3a,b). Paired comparisons between wild-type and $E p h B 1^{-1-}$ mutants for the mean number of BrdU-labeled cells in each region of the DG (SGZ, GL, H, and

ML) were performed using Student's $t$ test. The change in location of proliferating cells was most prominent in the SGZ and GL, where the EphB1 ${ }^{-1-}$ mice showed a significant decrease in number of proliferating cells in the SGZ ( $p=0.0002)$ and a concomitant increase in proliferation in the GL $(p=0.0078)$. Significant increases were also noted in the EphB1 ${ }^{-l-}$ mutants within the $\mathrm{H}$ $(p=0.0378)$ and the ML $(p=0.0048) . \mathrm{Ki}-67$ was used as an independent marker of proliferating cells and again indicated that proliferating cells are ectopically located outside the SGZ (Fig. $3 c)$. We also found a significant increase ( $p=0.0437$, Student's $t$ test) in the total number of proliferating $\mathrm{Ki}-67^{+}$cells found throughout the DG in EphB1 $1^{-/-}$animals $(122 \pm 15)$ when compared with wild-type animals $(72 \pm 8)$. Although the above methods provide only a "snapshot" of the ongoing proliferation in the DG, we have evidence that the majority of these proliferating cells are neurogenic progenitors. Independent confocal IF studies demonstrate that in wild-type and EphB1 $1^{-/-}$mice, $60-$ $70 \%$ of all $\mathrm{BrdU}^{+}$cells are also $\mathrm{DCX}^{+}$, whereas in separate experiments, $<20 \%$ are nestin/eGFP ${ }^{+}$and $\sim 25 \%$ are $\mathrm{GFAP}^{+}$ (supplemental Fig. 3, available at www.jneurosci.org as supplemental material). To further investigate neurogenesis, we administered three doses of BrdU separated by $2 \mathrm{~h}$ intervals and then assessed BrdU incorporation at multiple time points out to $28 \mathrm{~d}$. We found a significant increase ( $p<0.05$, Student's $t$ test) in the number of $\mathrm{BrdU}^{+}$cells in the DG of EphB1 ${ }^{-1-}$ animals (Fig. $3 d$ ). This increase in $\mathrm{BrdU}^{+}$cells corresponds to an increase in type II progenitors as implicated by the neonatal FACS comparisons shown in supplemental Figure 4 (available at www.jneurosci.org as supplemental material). Furthermore, at 7, 14, and $28 \mathrm{~d}$ after BrdU administration, there are more $\mathrm{DCX}^{+}$cells, but similar numbers of mature $\mathrm{NeuN}^{+}$neurons, carrying BrdU in the EphB1 ${ }^{-1-}$ animals (supplemental Table 1, available at www. jneurosci.org as supplemental material). Coexpression of other cell-specific markers along with BrdU, such as Iba-1 for microglia or CD45 for hematopoietic cells, was rarely seen (data not shown). The combined findings of decreased numbers of nestin/ $\mathrm{eGFP}^{+}$cells, increased numbers of proliferating neural progenitor cells, and ectopic mislocalized cells all indicate that loss of EphB1 protein by mutation leads to severe disruption of the normal program of neurogenesis in the adult hippocampus.

\section{EphB1 regulates cell polarity of immature neural progenitors in the DG}

Anti-DCX antibodies clearly label in the wild-type hippocampus a single immature apical dendritic outgrowth/process extending from each progenitor cell in the SGZ that projects radially through the GL toward the ML, where it then branches extensively in the ML to form the dense dendritic arbors observed in mature granule cell neurons (Figs. $1 b-d, 4 a$, left). However, in EphB1 $1^{-1-}$ mutants we observed that $\mathrm{DCX}^{+}$cellular outgrowths often exhibited premature branching within the GL (Fig. $4 a$, right). To quantify dendritic branching, equivalent $50 \mu \mathrm{m} \mathrm{sec}-$ tions of the hippocampus were processed for IF using anti-DCX antibodies to label cellular outgrowths, and $30 \mu \mathrm{m} z$-series confocal images were generated. Postimage analysis involved generating separate regions of interest (ROIs) in the $z$-plane in the GL and at three equally spaced positions within the ML (Fig. $4 b$ ). The number of $\mathrm{DCX}^{+}$signals that intersected these ROIs through the $z$-series was automatically counted. The total number of projections was then graphed relative to the total number of $\mathrm{DCX}^{+}$cell bodies within the same $z$-series (Fig. 4c). Paired comparisons of this relative dendritic complexity between wild-type and $E p h B 1^{-1-}$ mutants showed a significant increase within the GL 

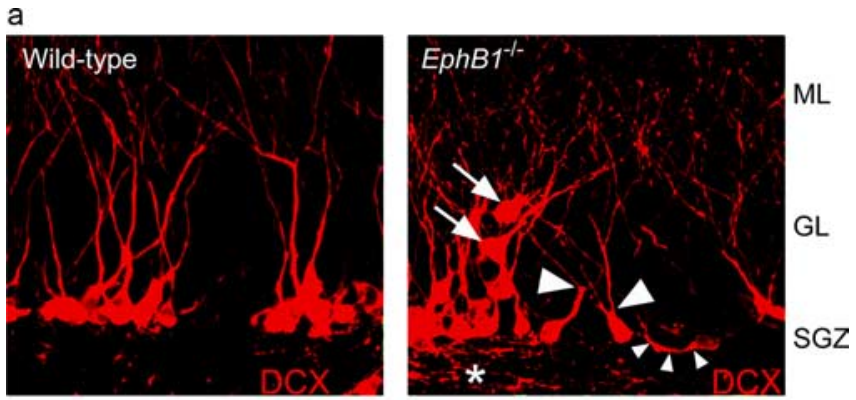

b

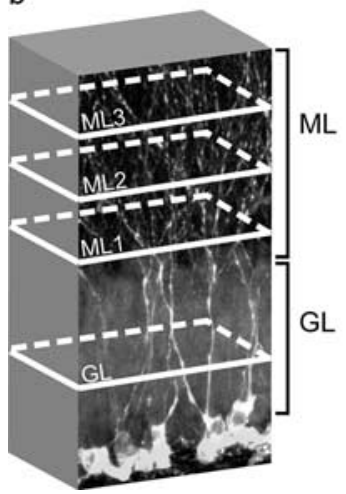

C

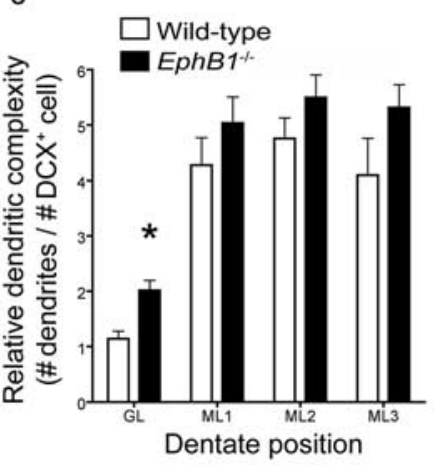

Figure 4. Premature branching and random polarization of the $D C X^{+}$dendritic outgrowth in adult mice lacking EphB1. $\boldsymbol{a}$, Confocal IF images of $\mathrm{DCX}{ }^{+}$cells (red) reveal in the EphB1 ${ }^{-1-}$ mutants premature branching of the dendritic outgrowth within the GL (large arrowheads), random nonradial orientation of the outgrowth (small arrowheads), and numerous cellular processes that encroach into the hilus (asterisk). Ectopic positioned cells are indicated (arrows). $\boldsymbol{b}$, Counts of $D C X^{+}$apical dendrites in the $G L$ and three different molecular layers (ML 1-3) involved the generation of ROls through the $z$-plane of $30 \mu \mathrm{m}$ sections and manual counting of $D C X^{+}$outgrowths within the ROl. Shown is a representative stacked confocal image of $D C X^{+}$ cells/processes from a wild-type brain. c, Consistent with premature branching, the number of dendritic projections per $\mathrm{DCX}{ }^{+}$cell in EphB1 ${ }^{-/-}$mutants is significantly increased from one (no branches) to two (one branch) in the $\mathrm{GL}$ of the dentate compared with wild-type specimens ( $p=0.0005$, Student's $t$ test). The graph represents the mean \pm SEM number of projections per DCX ${ }^{+}$cell at each of the ROls indicated in $\boldsymbol{b}$. A total of 270 wild-type and $248 \mathrm{EphB1}^{-/-}$ mutant processes were counted.

of EphB1 ${ }^{-1-}$ mutants ( $p=0.0005$, Student's $t$ test) and indicated that dendritic outgrowths from $\mathrm{DCX}^{+}$neural progenitors branch earlier in the EphB1 $1^{-1-}$ mutants as they project through the GL.

In addition to premature branching of apical dendritic outgrowths, $\mathrm{DCX}^{+}$cells in the EphB1 ${ }^{-1-}$ mutants, but not wild-type specimens, also often showed outgrowths that projected in a random, nonradial direction in relation to the GL as well as extensive basal labeling of cellular membranes that aberrantly extended into the hilus region (Fig. $4 a$ ). In total, the premature dendritic branching and presence of abnormal, apparently randomized cellular projections indicate that loss of EphB1 expression leads to severe disruption of the normal growth and polarity of cellular processes extending from hippocampal stem/progenitor cells.

\section{Ephrin-B3 is a key ligand for EphB1 in the hippocampal DG} Any discussion of a functional role for EphB1 in adult neurogenesis must include inspection of potential B-subclass ephrin ligands that may also participate in this process. Using mRNA in situ hybridization, we previously detected high levels of ephrin-B3 transcripts specifically in the GL of the DG and in CA1 neurons (Liebl et al., 2003). Because no reliable antibody probes are available, we analyzed ephrin-B3 protein localization in sections of animals carrying the ephrin-B $3^{\text {lacZ }}$ mutation that were reacted with X-gal (Fig. $5 a$ ) or processed for IF with anti- $\beta$-gal antibodies (Fig. $5 b$ ) to visualize the expression and subcellular localization of the ephrin-B3- $\beta$-gal fusion protein. Our generation and use of such "in-frame" lac $Z$ knock-in mutations to express $\beta$-galconjugated cytoplasmic-truncation proteins in the EphB2 (Henkemeyer et al., 1996), ephrin-B2 (Dravis et al., 2004), and ephrin-B3 (Yokoyama et al., 2001) genes has determined that the resulting fusion proteins recapitulate with high fidelity the expression patterns and subcellular localizations of the endogenous proteins. Here, with regards to ephrin-B3, we find very high levels of this protein concentrated within the DG specifically in the hilus and in the inner molecular layer (IML), with relatively lower levels in the outer molecular layer (OML). The distribution of ephrin-B3 in the hilus overlaps with calbindin staining, indicating that the protein is localized within the granule cell mossy fiber axons as they collect in the hilus and project to the CA3 neurons (data not shown), whereas the staining in the molecular layers is consistent with localization of this protein as well to the dendritic membranes of the mature granule cells. Although the high level of ephrin-B3 expression in the IML is similar to that seen for calretinin expressed in the axons projecting from mossy cells located in the hilus (supplemental Fig. 5a, available at www. jneurosci.org as supplemental material), closer IF examination of the GC-IML and the SGZ-H interfaces demonstrates that ephrin-B3 and calretinin are not expressed on the same cellular projections (supplemental Fig. 5b,c, available at www. jneurosci.org as supplemental material). Expression of ephrin-B2 using the ephrin- $B 2^{\text {lacZ }}$ allele indicates relatively weak expression of this EphB ligand in the adult DG, and in postnatal brains the expression appears mainly restricted to blood vessels (supplemental Fig. $1 c$, available at www.jneurosci.org as supplemental material). Finally, two independent expression databases, the Allen Brain Atlas of mRNA in situ (www.brainatlas.org) (Lein et al., 2007) and GENSAT of BAC-eGFP transgenic mice (www. gensat.org) (Gong et al., 2003) (supplemental Fig. 1c, available at www.jneurosci.org as supplemental material), both indicate that ephrin-B1 is expressed specifically in the SGZ.

Based on its high level of expression in the DG and availability of a protein-null mutation (Yokoyama et al., 2001), we assessed whether ephrin-B3 exhibits any ligand-like activity in regulating neurogenesis in the hippocampus. Confocal IF analysis indicates that $\mathrm{DCX}^{+}$stem/progenitor cells located in the SGZ sit directly on top of the high level of ephrin-B3 protein in the hilus where the mossy fibers accumulate and project to the CA3 region (Fig. $5 b$ ). Interestingly, dendritic branching and arborization of EphB1-expressing DCX ${ }^{+}$cells begins on the other side of the GL at the GL-ML junction, where the projecting apical directed dendritic outgrowth encounters the high expression of ephrin-B3 protein in the IML (Fig. 5b). Consistent with these expression/ subcellular localization patterns, we find that ephrin-B3 ${ }^{-1-}$ protein-null mutants phenocopy some of the defects observed above in the EphB1 ${ }^{-/-}$mutants. In particular, the ephrin-B3 ${ }^{-1-}$ mutants exhibit defective cell positioning of stem/progenitor cells outside of the SGZ (Fig. 5c) and can exhibit abnormal cellular processes extending into the hilus (Fig. $5 d$ ). Importantly, ectopic positioning of stem/progenitor cells or abnormal cellular processes penetrating the hilus were not generally observed in the ephrin-B3 ${ }^{\text {lacZ/lacZ }}$ homozygotes expressing the ephrin-B3- $\beta$-gal fusion protein (Fig. $5 b$ ), which can act as a functional ligand to bind Eph receptors and stimulate forward signaling. This indicates that loss of ephrin-B3 ligand-like activity is responsible for the defects observed in the ephrin-B3 $3^{-1-}$ null mutants. 
a

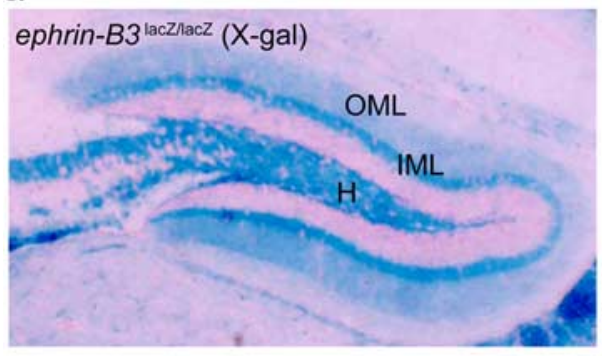

b
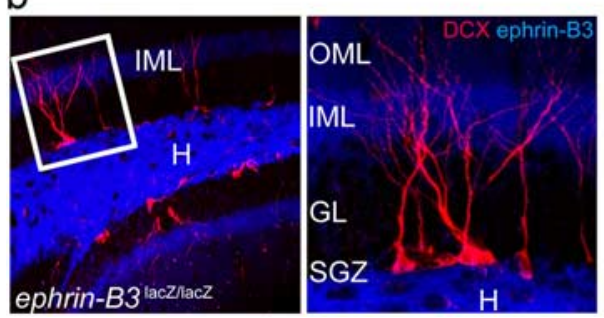

C

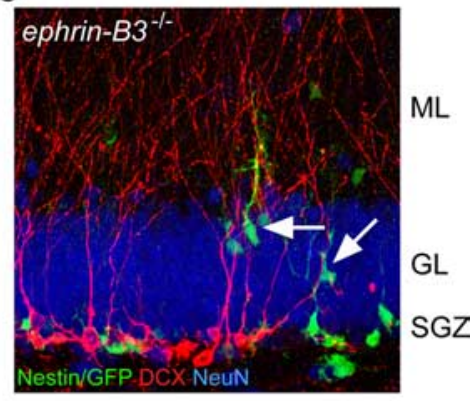

d

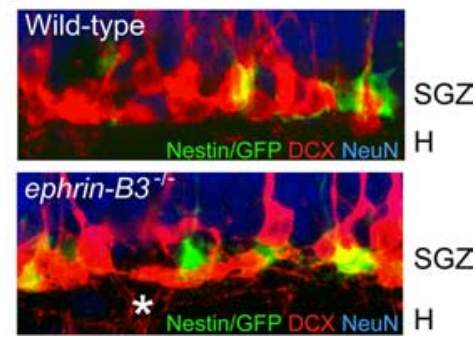

Figure 5. Ephrin-B3 exhibits ligand-like activity to control neural stem/progenitor positioning in the SGZ and prevent cellular processes from invading the hilus. $\boldsymbol{a}, X$-gal staining of an adult ephrin-B $3^{\text {lacZ/lacZ }}$ hippocampus demonstrates intense expression of the ephrin-B3- $\beta$-gal fusion protein (blue) in the $\mathrm{H}$ and IML, with relatively weaker labeling of the $0 \mathrm{ML}$. $\boldsymbol{b}$, Confocal IF for $\beta$-gal (blue) and $D C X$ (red) in the adult dentate of an ephrin- $B 3^{\text {lacZ/lacz }}$ homozygote shows intense expression of the ephrin-B3- $\beta$-gal fusion protein in the $H$ and IML, with little or no expression in the $S G Z$ and $G L$. Note that $D C X{ }^{+}$cells (red) primarily reside in the $S G Z$ and lie immediately adjacent to high levels of ephrin-B3 in the $\mathrm{H}$. High-magnification confocal $z$-series ( $30 \mu \mathrm{m}$ ) of boxed section indicates normal positioning of DCX ${ }^{+}$cells in the SGZ as well as normal morphology of the dendritic outgrowth in the ephrin$B 3^{\text {lacz/lacZ }}$ homozygote. C, Confocal IF for the nestin-eGFP transgene (green), DCX (red), and NeuN (blue) in the adult dentate of an

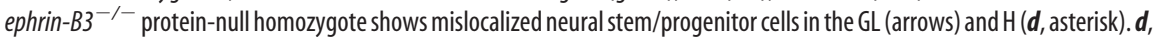
High-magnification confocal images of the SGZ in wild-type and ephrin- $B 3^{-/-}$adult animals demonstrate an increase in DCX ${ }^{+}$ basal cellular projections that penetrate the $\mathrm{H}$ (red) in the null mutant.

\section{EphB1 and EphB2 play coordinate roles in}

hippocampal development

Although the defects in hippocampal neurogenesis seen in the absence of EphB1 are interesting and suggestive of a role for Eph-ephrin interactions in this process, the absence of EphB1 has only minor visible effects on hippocampal morphology or size/number of mature granule cell neurons in the DG. We therefore asked whether another B-subclass Eph receptor might play a redundant, overlapping role in hippocampal neurogenesis. We performed RT-PCR analysis on isolated RNA from nestin/ $\mathrm{eGFP}^{+}$and nestin/eGFP ${ }^{-}$sorted populations of 2 -week-old microdissected dentates (Fig. 6a). The data demonstrate that EphB2 transcripts are expressed in the nestin/eGFP ${ }^{+}$cells to a degree similar to that found for EphB1 (Fig. 1f). This is also consistent with Allen Brain Atlas (www.brainatlas.org) mRNA in situ data for EphB2 expression (supplemental Fig. 1b, available at www. jneurosci.org as supplemental material). Furthermore, ephrin-B3 transcripts are expressed at high levels in the nestin/eGFP ${ }^{-}$pool (Fig. 6a), made up primarily of the mature granule cell neurons of the GL and a relatively small number of astrocytes, mossy cells, and microglia of the hilus, and these levels are consistent with the $\mathrm{X}$-gal staining and anti- $\beta$-gal immunoreactivity presented for ephrin-B3 above.

We then generated mice that carried the nestin-eGFP transgene and were compound-null for both EphB1 and EphB2 and noticed striking architectural abnormalities when compared with either the wild-type or the EphB1 ${ }^{-1-}$ null animals. Using Nisslstained serial coronal sections through wild-type, $E \mathrm{phB1} 1^{-1-}$, and $E p h B 1^{-1-} ; E p h B 2^{-1-}$ mutant brains, we found a significant dif- ference in the volume of the compound mutant DG only ( $p<0.0001$, ANOVA), suggesting a reduced number of mature granule cell neurons (Fig. 6b,c). Importantly, a planned comparison between wild-type and compound-null animals demonstrated a significant decrease $(p<$ 0.001 , Bonferroni corrected) in DG volume in the absence of both EphB1 and EphB2. Consistent with this, an even greater decrease in numbers of nestin/ $\mathrm{eGFP}^{+}$stem/progenitor cells was seen in the compound mutants when compared with the EphB1 ${ }^{-1-}$ animals (Fig. 6d). Defective lamination of the CA3 pyramidal neurons was also apparent and consistent with the coexpression of both EphB1 (Fig. $1 a$; supplemental Fig. $1 a$, available at www. jneurosci.org as supplemental material) and EphB2 (supplemental Fig. 1b, available at www.jneurosci.org as supplemental material) to this region of the hippocampus as well.

To determine whether EphB receptor forward signaling is important, we used the EphB2 lacZ allele, which expresses a cytoplasmic-truncated EphB2- $\beta$-gal fusion protein that is capable of acting as a ligand to bind ephrin-B molecules and stimulate reverse signaling, but cannot transduce forward signals into its own cell (Henkemeyer et al., 1996). We find that the resulting $E p h B 1^{-1-} ; E p h B 2^{\text {lacZ/lacZ }}$ compound mutants (Fig. $6 b, c$ ) present with a more severe reduction in the volume of the DG over that seen in the EphB1 $1^{-1-}$; EphB2 $2^{-1-}$ compound mutants $(p<0.05$, Bonferroni corrected), indicating a dominant-negative effect of the EphB2 truncation [see also Cowan et al. (2000), Hindges et al. (2002), and Dravis et al. (2004)]. Combined with the above results using ephrin-B3 ${ }^{-}$and ephrin-B3 ${ }^{\text {lacZ }}$ mutations, our data provide strong evidence that EphB-mediated forward signaling plays a key role in neurogenesis within the DG.

\section{Discussion}

Molecular cues governing hippocampal neurogenesis, both postnatal and adult, are only beginning to be revealed. A number of recent studies have demonstrated that Eph receptor tyrosine kinases and their ephrin ligands are present in the hippocampus and have implicated that their bidirectional signals regulate various aspects of synapse formation, maturation, and function (Murai and Pasquale, 2004; Martinez and Soriano, 2005). Here we provide the first evidence that EphB1 is expressed in the neuronal progenitor pool of the hippocampal DG. More importantly, we demonstrate that the genetic ablation of EphB1 impairs several aspects of neurogenesis in the hippocampus, leading to a reduction in the number of nestin ${ }^{+}$stem cells, dysregulation of proliferation and cell body positioning, and abnormal branching/polarity of cellular outgrowths. We also propose ephrin-B3, expressed on the axons and dendrites of the granule cell neurons, as a primary ligand for EphB1, because deletion of this ligand recapitulates several of the defects seen in the EphB ${ }^{-1-}$ mutants. Finally, we propose that EphB2, also expressed in the nestin/ 
$\mathrm{eGFP}^{+}$progenitor pool, may play a redundant role with EphB1 in regulating at least some aspects of neurogenesis within the DG.

Recent studies of other molecular signals involved in hippocampal neurogenesis have suggested that fibroblast growth factor receptor 1 (Fgfr1), Wnt, and Sonic hedgehog (Shh) signaling pathways actively regulate neurogenesis within the DG. Specific deletion of the Fgfr 1 from the radial glial-like neuronal progenitors resulted in severe defects in hippocampal neurogenesis (Ohkubo et al., 2004). Mice with Wnt mutations or lacking key Wnt signaling components present with major defects in neural progenitor cell proliferation and abnormal morphogenesis in the hippocampus (Zhou et al., 2004; Lie et al., 2005). It was discovered that Wnt 3 is produced in the hilus, likely by the GFAP ${ }^{+}$ radial glial-like cells that are the putative quiescent stem cells of the SGZ. Similarly, Shh has been shown to regulate proliferation in the adult hippocampus (Lai et al., 2003) and was later found to target the $\mathrm{GFAP}^{+}$neural progenitor population (Ahn and Joyner, 2005). We now show that EphB receptor forward signaling should be added to this list of molecules that regulate neurogenesis in the hippocampus.

Our observation of increased cell proliferation in the hippocampus of EphB1 $1^{-1-}$ mutants is consistent with the report by Ricard et al. (2006) that showed that a loss of ephrin-B3 led to increases in the number of proliferating cells located in a different neurogenic region, the subventricular zone (SVZ). However, although Ricard et al. (2006) noted an increase in SVZ proliferation in the absence of ephrinB3, they did not specifically identify which Eph receptor was involved in this niche. Using the nestin-eGFP reporter, we were able to demonstrate that in the hippocampus the EphB1 and EphB2 receptors are coexpressed on early type I and type IIa neural progenitors. Another key finding of our study is that this increase in proliferation observed in the absence of EphB1 occurs despite significant reductions in the actual number of early neural progenitors. Combined with Ricard et al. (2006), it appears that Eph-ephrin bidirectional signaling negatively regulates cell proliferation in both neural stem cell niches. Interestingly, this interpretation seems in direct opposition to our previous findings in the intestinal stem/progenitor cell niche (Holmberg et al., 2006), in which EphB2 and EphB3 were shown to work in concert to promote cell cycle reentry. Genetic elimination of Eph receptor expression or transient in vivo blockage of Eph-ephrin interactions resulted in decreased proliferation and an altered architecture of the intestinal niche. One way to connect these two apparently disparate roles for EphB receptors in stem cells is to propose that EphB1 may regulate self-renewal in the type I cells in a manner similar to its function in the intestinal crypt. However, if this were the case, we would expect to see the eventual elimination of this precursor pool over time. We do not see this. Furthermore, we find that the approximate number of these nestin/eGFP ${ }^{+}$ mutant. b

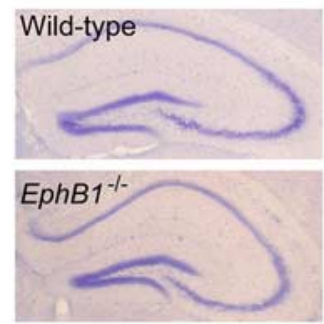

d

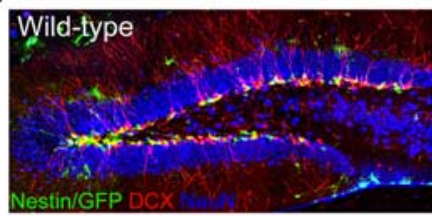

DG

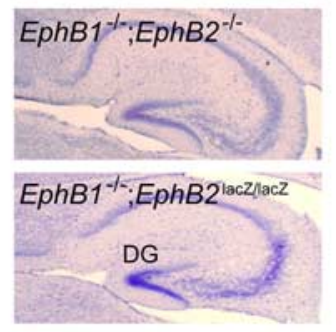

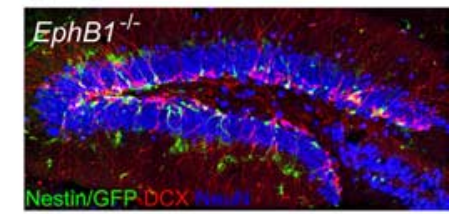

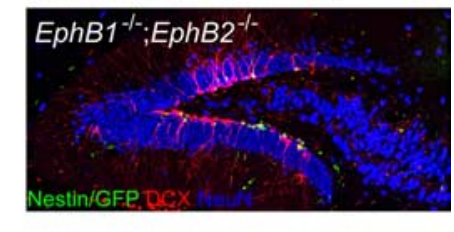

Figure 6. Combined loss of EphB1 and EphB2 results in even more dramatic neurogenic defects in the dentate gyrus. $\boldsymbol{a}, \mathrm{RT}-\mathrm{PCR}$ from total RNA collected from 2-week-old microdissected dentate cells containing the nestin-eGFP transgene and sorted for eGFP fluorescence demonstrates that EphB2 transcripts are found specifically in the GFP ${ }^{+}$stem/progenitor population. In contrast, cytes in the hilus. $\boldsymbol{b}$, Nissl stains of hippocampi from indicated adult mice demonstrate that the EphB1 ${ }^{-1-} ;$ EphB2 $^{-1-} \mathrm{com}^{-}$ 列 single mutant. c, Estimated DG volume for wild-type, EphB1 $1^{-/-}$, EphB1 ${ }^{-1-}$;EphB2 compound null and more dramatic decrease in the numbers of stem/progenitor cells as well as mature granule cell neurons than the $E p h B 1^{-1-}$ single

progenitors in the absence of EphB1 is generally $50 \%$ of that seen in the wild-type DG at all time points tested, including at early postnatal stages and in aged adults. In addition, the genetic ablation of EphB1 and EphB2 together still does not result in the exhaustion of the type I progenitors, even at 12 months of age. How then can Eph-ephrin bidirectional signals both promote and inhibit proliferation?

We instead hypothesize that EphB1 plays a dual role in development/maintenance of the hippocampal DG. First, initial migration of neural progenitors into the hippocampal primordium is at least partially regulated by EphB1, suggested by the substantial reduction in the early progenitor pool at postnatal day 7 in the absence of this receptor. It is also possible that the role of EphB1 in this altered migration is the mechanism that underlies the ectopic localization of neural progenitors outside of the SGZ in the adult $E p h B 1^{-1-}$ mutant. Second, within the normal homeostatic mechanisms that regulate neurogenesis in the DG, EphB1 acts either to accelerate cell cycle progression in the type II cells or to induce cell cycle exit of these cells. Similar control of neurogenesis has been demonstrated for the cell cycle regulator p27Kip1 (Doetsch et al., 2002). Genetic elimination of p27Kip1 results in an increase in the type $\mathrm{C}$ transit-amplifying cells of the SVZ, a population very similar to the type II cells of the SGZ.

One of the striking phenotypes uncovered in the absence of EphB1 forward signaling is the disruption of the normal architecture of the SGZ neurogenic niche. In the absence of EphB1 receptor signaling activity or ephrin-B3 ligand activity, we find 
that elements of the neural progenitor pool are mislocalized outside of the SGZ and into the GL, ML, and hilus. Furthermore, the $\mathrm{DCX}^{+}$progenitors seem to have an altered cellular polarity, in that they produce dendritic protrusions and branching in somewhat random directions. It is intriguing to speculate that altered cell polarity could explain the increase in proliferation seen in the absence of EphB1, an idea not without precedent. Deletion of the mouse Lgl1 protein, a homolog of Drosophila Lethal (2) giant larvae ( $\mathrm{Lgl})$, results in a disruption of cell polarity and a neoplastic transformation of neuroepithelial cells (Klezovitch et al., 2004). In fact, mutations in three Drosophila genes, scribble (scrib), discs-large (dlg), and lethal giant larvae (lgl), cause simultaneous loss of polarity and overproliferation (Bilder, 2004). Interestingly, human Scribble has been linked to the activation of the small Rho-GTPase cdc42 (Dow et al., 2007). Recently, it has been demonstrated that cdc42 regulates neural progenitor fate at the apical surface and its deletion results in the loss of the selfrenewing neural progenitors and a fate conversion to the symmetrically dividing progenitor pool (Cappello et al., 2006). Considering that both EphA and EphB proteins have also been linked to cdc42 (Noren and Pasquale, 2004), we speculate that loss of EphB receptors may disrupt cell polarity, perhaps through cdc42, and produce the defects observed after disruption of forward signaling.

One of the important challenges of analyzing any DG phenotype that involves a developmental component is to uncover whether the adult phenotype is simply a remnant of an earlier developmental defect. A simple explanation for our data is that in the absence of EphB1, a developmental defect in migration and neurogenesis occurs in the late embryonic and early postnatal formation of the hippocampus, which remains throughout adulthood. Although such an explanation is certainly plausible, it does not necessarily exclude a role for EphB1 in the adult hippocampus. And, although the early hippocampus of the $E p h B 1^{-/-}$mutant is seeded with significantly fewer stem/progenitors during its early formation, enough homeostatic mechanisms are still in place to allow for the development of a mostly normal hippocampus based on size and cellularity. It is likely that the increased proliferation we report in the $E p h B 1^{-/-}$mutant is the reason that fewer progenitor cells are capable of producing nearly normal numbers of mature GC neurons and thereby creating a normal sized DG. However, the only way to truly analyze the function of EphB1 in adult neurogenesis is through the conditional elimination of this gene from the adult hippocampal progenitor pool.

It is important to recognize that other A- and B-subclass Eph receptors and ephrin ligands may also aid in patterning the SGZ neurogenic niche. For example, ephrin-B1 is expressed in the SGZ based on BAC-eGFP data, and ephrin-B2 is likely expressed in the microvasculature in close proximity to the neurogenic niche (supplemental Fig. 1, available at www. jneurosci.org as supplemental material). However, we present here a definitive role for EphB1 in neurogenesis within the hippocampal DG. Genetic elimination of the EphB1 receptor causes alterations in progenitor cell migration, cell cycle progression, and cell polarity. We further introduce an additional role for EphB2 in this process. Finally, we present compelling data to suggest that the interaction of these EphB receptors with the ephrin-B3 ligand within the architecture of the DG is what establishes the normal pattern and localization of the SGZ neurogenic niche.

\section{References}

Abrous DN, Koehl M, Le Moal M (2005) Adult neurogenesis: from precursors to network and physiology. Physiol Rev 85:523-569.

Ahn S, Joyner AL (2005) In vivo analysis of quiescent adult neural stem cells responding to Sonic hedgehog. Nature 437:894-897.

Bilder D (2004) Epithelial polarity and proliferation control: links from the Drosophila neoplastic tumor suppressors. Genes Dev 18:1909-1925.

Brambilla R, Schnapp A, Casagranada F, Labrador JP, Bergemann AD, Flanagan JG, Pasquale EB, Klein R (1995) Membrane-bound LERK2 ligand can signal through three different Eph-related receptor tyrosine kinases. EMBO J 14:3116-3126.

Cappello S, Attardo A, Wu X, Iwasato T, Itohara S, Wilsch-Brauninger M, Eilken HM, Rieger MA, Schroeder TT, Huttner WB, Brakebusch C, Gotz M (2006) The Rho-GTPase cdc42 regulates neural progenitor fate at the apical surface. Nat Neurosci 9:1099-1107.

Conover JC, Doetsch F, Garcia-Verdugo JM, Gale NW, Yancopoulos GD, Alvarez-Buylla A (2000) Disruption of Eph/ephrin signaling affects migration and proliferation in the adult subventricular zone. Nat Neurosci 3:1091-1097.

Cowan CA, Yokoyama N, Bianchi LM, Henkemeyer M, Fritzsch B (2000) EphB2 guides axons at the midline and is necessary for normal vestibular function. Neuron 26:417-430.

Davis S, Gale NW, Aldrich TH, Maisonpierre PC, Lhotak V, Pawson T, Goldfarb M, Yancopoulos GD (1994) Ligands for EPH-related receptor tyrosine kinases that require membrane attachment or clustering for activity. Science 266:816-819.

Depaepe V, Suarez-Gonzalez N, Dufour A, Passante L, Gorski JA, Jones KR, Ledent C, Vanderhaeghen P (2005) Ephrin signalling controls brain size by regulating apoptosis of neural progenitors. Nature 435:1244-1250.

Doetsch F, Verdugo JMG, Caille I, Alvarez-Buylla A, Chao MV, CasacciaBonnefil P (2002) Lack of the cell-cycle inhibitor p27Kip1 results in selective increase of transit-amplifying cells for adult neurogenesis. J Neurosci 22:2255-2264.

Dow LE, Kauffman JS, Caddy J, Peterson AS, Jane SM, Russell SM, Humbert PO (2007) The tumour-suppressor Scribble dictates cell polarity during directed epithelial migration: regulation of Rho GTPase recruitment to the leading edge. Oncogene 26:2272-2282.

Dravis C, Yokoyama N, Chumley MJ, Cowan CA, Silvany RE, Shay J, Baker LA, Henkemeyer M (2004) Bidirectional signaling mediated by ephrin-B2 and EphB2 controls urorectal development. Dev Biol 271:272-290.

Esposito MS, Piatti VC, Laplagne DA, Morgenstern NA, Ferrari CC, Pitossi FJ, Schinder AF (2005) Neuronal differentiation in the adult hippocampus recapitulates embryonic development. J Neurosci 25:10074-10086.

Gale NW, Holland SJ, Valenzuela DM, Flenniken A, Pan L, Ryan TE, Henkemeyer M, Strebhardt K, Hirai H, Wilkinson DG, Pawson T, Davis S, Yancopoulos GD (1996) Eph receptors and ligands comprise two major specificity subclasses and are reciprocally compartmentalized during embryogenesis. Neuron 17:9-19.

Ge S, Goh EL, Sailor KA, Kitabatake Y, Ming GL, Song H (2006) GABA regulates synaptic integration of newly generated neurons in the adult brain. Nature 439:589-593.

Gong S, Zheng C, Doughty ML, Losos K, Didkovsky N, Schambra UB, Nowak NJ, Joyner A, Leblanc G, Hatten ME, Heintz N (2003) A gene expression atlas of the central nervous system based on bacterial artificial chromosomes. Nature 425:917-925.

Henkemeyer M, Orioli D, Henderson JT, Saxton TM, Roder J, Pawson T, Klein R (1996) Nuk controls pathfinding of commissural axons in the mammalian central nervous system. Cell 86:35-46.

Himanen JP, Chumley MJ, Lackmann M, Li C, Barton WA, Jeffrey PD, Vearing C, Geleick D, Feldheim DA, Boyd AW, Henkemeyer M, Nikolov DB (2004) Repelling class discrimination: ephrin-A5 binds to and activates EphB2 receptor signaling. Nat Neurosci 7:501-509.

Hindges R, McLaughlin T, Genoud N, Henkemeyer M, O’Leary DD (2002) EphB forward signaling controls directional branch extension and arborization required for dorsal-ventral retinotopic mapping. Neuron 35:475-487.

Holland SJ, Gale NW, Mbamalu G, Yancopoulos GD, Henkemeyer M, Pawson T (1996) Bidirectional signaling through the Eph family receptor Nuk and its transmembrane ligands. Nature 383:722-725.

Holmberg J, Frisen J (2002) Ephrins are not only unattractive. Trends Neurosci 25:239-243 
Holmberg J, Genander M, Halford MM, Anneren C, Sondell M, Chumley MJ, Silvany RE, Henkemeyer M, Frisen J (2006) EphB receptors coordinate migration and proliferation in the intestinal stem cell niche. Cell 125:1151-1163.

Holscher C (2003) Time, space and hippocampal functions. Rev Neurosci 14:253-284.

Kee N, Teixeira CM, Wang AH, Frankland PW (2007) Preferential incorporation of adult-generated granule cells into spatial memory networks in the dentate gyrus. Nat Neurosci 10:355-362.

Klezovitch O, Fernandez TE, Tapscott SJ, Vasioukhin V (2004) Loss of cell polarity causes severe brain dysplasia in Lgll knockout mice. Genes Dev 18:559-571.

Lai K, Kaspar BK, Gage FH, Schaffer DV (2003) Sonic hedgehog regulates adult neural progenitor proliferation in vitro and in vivo. Nat Neurosci 6:21-27.

Lein ES, Hawrylycz MJ, Ao N, Ayres M, Bensinger A, Bernard A, Boe AF, Boguski MS, Brockway KS, Byrnes EJ, Chen L, Chen L, Chen TM, Chin MC, Chong J, Crook BE, Czaplinska A, Dang CN, Datta S, Dee NR, et al (2007) Genome-wide atlas of gene expression in the adult mouse brain. Nature 445:168-176.

Lennington JB, Yang Z, Conover JC (2003) Neural stem cells and the regulation of adult neurogenesis. Reprod Biol Endocrinol 1:99.

Lie DC, Colamarino SA, Song HJ, Desire L, Mira H, Consiglio A, Lein ES, Jessberger S, Lansford H, Dearie AR, Gage FH (2005) Wnt signalling regulates adult hippocampal neurogenesis. Nature 437:1370-1375.

Liebl DJ, Morris CJ, Henkemeyer M, Parada LF (2003) mRNA expression of ephrins and Eph receptor tyrosine kinases in the neonatal and adult mouse central nervous system. J Neurosci Res 71:7-22.

Lledo PM, Alonso M, Grubb MS (2006) Adult neurogenesis and functional plasticity in neuronal circuits. Nat Rev Neurosci 7:179-193.

Martinez A, Soriano E (2005) Functions of ephrin/Eph interactions in the development of the nervous system: emphasis on the hippocampal system. Brain Res Brain Res Rev 49:211-226.

Ming GL, Song H (2005) Adult neurogenesis in the mammalian central nervous system. Annu Rev Neurosci 28:223-250.

Murai KK, Pasquale EB (2004) Eph receptors, ephrins, and synaptic function. Neuroscientist 10:304-314.

Noren NK, Pasquale EB (2004) Eph receptor-ephrin bidirectional signals that target Ras and Rho proteins. Cell Signal 16:655-666.
Ohkubo Y, Uchida AO, Shin D, Partanen J, Vaccarino FM (2004) Fibroblast growth factor receptor 1 is required for the proliferation of hippocampal progenitor cells and for hippocampal growth in mouse. J Neurosci 24:6057-6069.

Overstreet-Wadiche LS, Westbrook GL (2006) Functional maturation of adult-generated granule cells. Hippocampus 16:208-215.

Phelps EA (2004) Human emotion and memory: interactions of the amygdala and hippocampal complex. Curr Opin Neurobiol 14:198-202.

Ricard J, Salinas J, Garcia L, Liebl DJ (2006) EphrinB3 regulates cell proliferation and survival in adult neurogenesis. Mol Cell Neurosci 31:713-722.

Richardson MP, Strange BA, Dolan RJ (2004) Encoding of emotional memories depends on amygdala and hippocampus and their interactions. Nat Neurosci 7:278-285.

Song H, Kempermann G, Overstreet Wadiche L, Zhao C, Schinder AF, Bischofberger J (2005) New neurons in the adult mammalian brain: synaptogenesis and functional integration. J Neurosci 25:10366-10368.

Squire LR (1992) Memory and the hippocampus: a synthesis from findings with rats, monkeys, and humans. Psychol Rev 99:195-231.

Suzuki WA (2006) Encoding new episodes and making them stick. Neuron 50:19-21.

Tozuka Y, Fukuda S, Namba T, Seki T, Hisatsune T (2005) GABAergic excitation promotes neuronal differentiation in adult hippocampal progenitor cells. Neuron 47:803-815.

Wang H, Chia W (2005) Drosophila neural progenitor polarity and asymmetric division. Biol Cell 97:63-74.

Williams SE, Mann F, Erskine L, Sakurai T, Wei S, Rossi DJ, Gale NW, Holt CE, Mason CA, Henkemeyer M (2003) Ephrin-B2 and EphB1 mediate retinal axon divergence at the optic chiasm. Neuron 39:919-935.

Yokoyama N, Romero MI, Cowan CA, Galvan P, Helmbacher F, Charnay P, Parada LF, Henkemeyer M (2001) Forward signaling mediated by ephrin-B3 prevents contralateral corticospinal axons from recrossing the spinal cord midline. Neuron 29:85-97.

Yu TS, Dandekar M, Monteggia LM, Parada LF, Kernie SG (2005) Temporally regulated expression of Cre recombinase in neural stem cells. Genesis 41:147-153.

Zhou CJ, Zhao C, Pleasure SJ (2004) Wnt signaling mutants have decreased dentate granule cell production and radial glial scaffolding abnormalities. J Neurosci 24:121-126. 